\title{
New Light on the Objective Indefiniteness or Literal Interpretation of Quantum Mechanics
}

\author{
David Ellerman \\ University of California Riverside \\ University of Ljubljana, Slovenia
}

December 27, 2018

\begin{abstract}
The development of the new logic of partitions (= equivalence relations) dual to the usual Boolean logic of subsets, and its quantitative version as the new logical theory of information provide the basic mathematical concepts to describe distinctions/indistinctions, definiteness/indefiniteness, and distinguishability/indistinguishability. They throw some new light on the objective indefiniteness or literal interpretation of quantum mechanics (QM) advocated by Abner Shimony. This paper shows how the mathematics of QM is the math of indefiniteness and thus, literally and realistically interpreted, it describes an objectively indefinite reality at the quantum level. In particular, the mathematics of wave propagation is shown to also be the math of the evolution of indefinite states that do not change the degree of indistinctness between states. This corrects the historical wrong turn of seeing QM as "wave mechanics" rather than the mechanics of particles with indefinite/definite properties. For example, the so-called "wave-particle duality' for particles is the juxtaposition of the evolution of a particle having an indefinite position ("wave-like" behavior) with a particle having a definite position (particle-like behavior).
\end{abstract}

\section{Contents}

1 Introduction 2

2 Partition logic and logical information theory 2

3 What is a superposition state? 4

3.1 The two classical notions of abstraction . . . . . . . . . . . . . 4

3.2 The general notion of $\# 2$ abstraction $\ldots \ldots \ldots \ldots \ldots \ldots \ldots$

3.3 From incidence to density matrices . . . . . . . . . . . . . . . . . . 6

3.4 Density matrices in quantum mechanics . . . . . . . . . . . . . . . . 6

4 Interpreting the inner product 7

4.1 The classical case . . . . . . . . . . . . . . . . . . . . 7



5 Numerical attributes and measurement 8

5.1 The classical case . . . . . . . . . . . . . . . . . . . 8

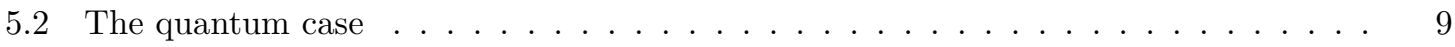


6 Logical information theory at the classical and quantum level 10

6.1 The classical case . . . . . . . . . . . . . . . . . . . . . . . . 10

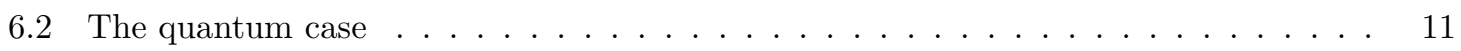

7 Quantum dynamics and measurement 12

7.1 Von Neumann's type 2 processes . . . . . . . . . . . . . . . . . . . 12

7.2 Von Neumann's type 1 processes $\ldots \ldots \ldots \ldots \ldots \ldots \ldots \ldots$

8 Group theory and QM 15

9 Concluding remark

\section{Introduction}

New developments in mathematical logic and the related logical information theory have helped to further elucidate what Abner Shimony advocated as the objective indefiniteness or literal interpretation of quantum mechanics (QM).

From these two basic ideas alone - indefiniteness and the superposition principle - it should be clear already that quantum mechanics conflicts sharply with common sense. If the quantum state of a system is a complete description of the system, then a quantity that has an indefinite value in that quantum state is objectively indefinite; its value is not merely unknown by the scientist who seeks to describe the system. Furthermore, since the outcome of a measurement of an objectively indefinite quantity is not determined by the quantum state, and yet the quantum state is the complete bearer of information about the system, the outcome is strictly a matter of objective chance - not just a matter of chance in the sense of unpredictability by the scientist. Finally, the probability of each possible outcome of the measurement is an objective probability. [27, p. 47]

These statements ... may collectively be called "the Literal Interpretation" of quantum mechanics. This is the interpretation resulting from taking the formalism of quantum mechanics literally, as giving a representation of physical properties themselves, rather than of human knowledge of them, and by taking this representation to be complete. 28, pp. 6-7]

The same theme has been continued by Shimony's student and colleague, Gregg Jaeger.

The conceptual elements of quantum theory that now underlie our picture of the physical world include objective chance, quantum interference, and the objective indefiniteness of dynamical quantities. Quantum interference, which is directly observable, was readily absorbed by the physics community. Objective chance and indefiniteness, being of more philosophical significance, gained acceptance only after much debate and conceptual analysis, when it was recognized that observed phenomena are better understood through these notions than through older ones or hidden variables. 24, p. vii]

Since the elucidation of this interpretation of QM pivots on the notions of indefiniteness and indistinguishability, it will be called the objective indefiniteness (OI) interpretation.

\section{Partition logic and logical information theory}

In the past, the most basic form of logic was the Boolean logic of subsets (usually called "propositional" logic), but from the mathematical point of view, it is only half of logic. The notion of a 
subset has a category-theoretic dual in the notion of a quotient set, equivalence relation, or partition (three equivalent notions). With some anticipation in the work of Gian-Carlo Rota 21, the logic of partitions was developed for arbitrary partitions on a set ([11; [12])-so that, at the mathematical level, partition logic is equally fundamental as the logic of the dual notion, the usual Boolean logic of subsets.

A partition $\pi=\left\{B_{1}, \ldots, B_{m}\right\}$ on a set $U=\left\{u_{1}, \ldots, u_{n}\right\}$ is a set of non-empty subsets or blocks $B_{j} \subseteq U$ that are disjoint and whose union is $U 1$ A (real-valued) numerical attribute on $U$ is a function $f: U \rightarrow \mathbb{R}$. It has certain numerical values, say $\left\{r_{1}, \ldots, r_{m}\right\}$, and its inverse-image is a partition $\pi=\left\{f^{-1}\left(r_{j}\right)\right\}_{j=1, \ldots, m}$ on $U$ with blocks $B_{j}=f^{-1}\left(r_{j}\right)$. Partitions are important for the objective indefiniteness interpretation of QM because they explicate the notions of indistinction (or indefiniteness or indistinguishability) and distinction at the logical level. Two elements $u, u^{\prime} \in B_{j}=$ $f^{-1}\left(r_{j}\right)$ are indistinct in terms of the attribute $f$ or, equivalently, the partition $\pi$, and two elements of $U$ in different blocks are distinct in terms of $f$ or $\pi$.

In view of the parallelism between subset logic and partition logic, each has a quantitative version for finite $U$. The normalized number of elements $\frac{|S|}{|U|}$ of a subset $S \subseteq U$ is the (LaplaceBoole) probability of the event $S$ [5]. A ordered pair $\left(u, u^{\prime}\right)$ of elements that are indistinct in $\pi$ is an indistinction or indit of $\pi$, and the set of indits of a partition is its indit-set indit $(\pi)$-which is just the equivalence relation indit $(\pi) \subseteq U \times U$ associated with the partition $\pi$. Similarly an ordered pair $\left(u, u^{\prime}\right)$ of elements in different blocks of $\pi$ is a distinction or dit of $\pi$, and the set of dits of a partition is its dit-set $\operatorname{dit}(\pi)=U \times U-\operatorname{indit}(\pi)$ which is called an apartness relation or partition relation.

The partition logic analogue of the normalized number of elements $\frac{|S|}{|U|}$ is the normalized number of dits $\frac{|\operatorname{dit}(\pi)|}{|U \times U|}$ of a partition $\pi$ which is the logical entropy of the partition $\pi$ [10. Thus the dual to the logical (i.e., finite discrete) probability theory that arises as the quantitative version of subset logic is the logical theory of information that arises as the quantitative version of partition logic 2 Logical information theory is the foundational theory of information based on the intuitive idea of information as distinctions, differences, and distinguishability [14. All the usual definitions of simple, joint, conditional, and mutual Shannon entropy are obtained by a uniform (dit to bit) transformation of the corresponding definitions for logical entropy. The intuitive idea is that instead of counting the number of distinctions, the Shannon entropy counts the (average minimum) number of letters in a binary code (bits) it takes to make the same distinctions (i.e., to uniquely encode the distinct messages), so the Shannon theory is repositioned as the specialized theory about coding and communications [25].

Our purpose is to show how these new developments in mathematical logic and information theory elucidate the objective indefiniteness interpretation of QM. Classically, reality was thought to be "definite all the way down." But QM gives a different message-that reality is (objectively) indefinite at the quantum level. The problem is that we have little idea how to intuitively imagine such a reality and hence the problem of building a realistic interpretation of QM that goes beyond the bare mathematical formalism. Since the development of QM in the first quarter of the twentieth century, interpretations have multiplied rather than converged which indicates the difficulty of the problem. But if quantum reality is objectively indefinite, then this new mathematics built on the notions of distinction/indistinction, definiteness/indefiniteness, and distinguishability/indistinguishability, will provide some important tools to elucidate that reality.

\footnotetext{
${ }^{1}$ Partition logic works with arbitrary sets but, for expository purposes, we restrict ourselves here to finite sets and finite dimensional Hilbert spaces.

${ }^{2}$ Both logical probability and logical entropy have obvious generalizations when the points of $U$ have probabilties assigned to them instead of being equiprobable.
} 


\section{What is a superposition state?}

\subsection{The two classical notions of abstraction}

The strategy of elucidation is to consider certain classical concepts, such as a set of elements $S \subseteq U$ which could be a subset $S \subseteq B$ of a block in a partition, and then to show how its features are vastly generalized in the the corresponding quantum concept, such as a superposition state.

To understand the classical version of a superposition state, we need to consider the abstraction principle which is most clearly understood in mathematics. If $\sim$ is an equivalence relation on $U$ and $A(u)$ is "the abstraction from $u$ ", then the abstraction principle turns equivalence into identity:

$$
A(u)=A\left(u^{\prime}\right) \text { iff } u \sim u^{\prime}
$$

for all $u, u^{\prime} \in U$. A well-known example of an abstraction principle is Frege's "direction principle" which Stewart Shapiro described as: for any lines $l_{1}$ and $l_{2}$ in some domain, the "direction of $l_{1}$ is identical to the direction of $l_{2}$ if and only if $l_{1}$ is parallel to $l_{2}$." [26, p. 107] Abstraction turns equivalence of being parallel into the identity of direction. But there are two different ways for this abstraction principle to be satisfied. The version often used by the proverbial 'working mathematician' will be called the \#1 abstraction, namely, just the equivalence class, e.g., a block in the partition of a set of lines in a plane where the equivalence is " $l_{1}$ is parallel to $l_{2}$ ". If $[l]$ is the parallelism equivalence class of the line $l$, then the abstraction principle of turning equivalence into identity is clearly satisfied: $l_{1} \simeq l_{2}$ iff $\left[l_{1}\right]=\left[l_{2}\right]$ (where $\simeq$ is the equivalence relation of being parallel).

But there is a second way to interpret abstraction and that is the one relevant to understanding superposition in QM. It will be referred to as the \#2 type of abstraction where the "the direction of $l$ " is an abstract object that is definite on what is common to parallel lines (i.e., their direction) but abstracts away from where they differ, i.e., is indefinite on how they differ.

Within mathematics, the \#2 type of abstraction is highlighted by the recent development of homotopy type theory. There is an equivalence relation $A \simeq B$ between topological spaces which is realized by a continuous map $f: A \rightarrow B$ such that there is an inverse $g: B \rightarrow A$ so the $f g: B \rightarrow B$ is homotopic to $1_{B}$ (i.e., can be continuously deformed in $1_{B}$ ) and $g f$ is homotopic to $1_{A}$. According to the 'classical' homotopy theorist, Hans-Joachim Baues, "Homotopy types are the equivalence classes of spaces" 3. under this equivalence relation. That is the \#1 type of abstraction.

But the interpretation offered in homotopy type theory (HoTT) is expanding identity to "coincide with the (unchanged) notion of equivalence" in the words of the Univalent Foundations Program [31, p. 5] so it would refer to the \#2 homotopy type, i.e., 'the homotopy type' that is definite on the mathematical properties shared by all spaces in an equivalence class of homotopic spaces (but is indefinite on the differences). Expanding identity to coincide with equivalence is another way to describe the \#2 abstracting from the class $S$ of equivalent entities to the abstract entity that is definite on what is common to the elements $u \in S$ but is indefinite on where they differ.

For instance, 'the homotopy type' is not one of the classical topological spaces (with points etc.) in the \#1 equivalence class of homotopic spaces-just as Frege's \#2 abstraction of direction is not among the lines in the equivalence class of parallel lines with the same direction.

While classical homotopy theory is analytic (spaces and paths are made of points), homotopy type theory is synthetic: points, paths, and paths between paths are basic, indivisible, primitive notions. [31, p. 59]

Consider the homotopy example of 'the path going once (clockwise) around the hole' in an annulus $A$ (disk with one hole as in Figure 1), i.e., the abstract entity 1 in the fundamental group $\pi_{0}(A)$ of the annulus: $1 \in \pi_{0}(A) \cong \mathbb{Z}$ : 


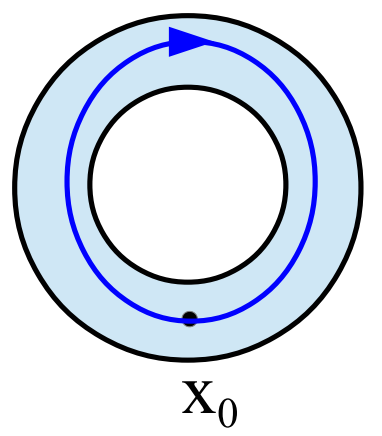

Figure 1: 'the path going once (clockwise) around the hole'

Note that 'the path going once (clockwise) around the hole' has the definite property of "going once (clockwise) around the hole" but is indefinite on any of the particular (coordinatized) paths that constitute the equivalence class of coordinatized once-around paths deformable into one another.

In a similar manner, we can view other common \#2 abstractions such as: 'the cardinal number 5' that captures what is common to the isomorphism class of all five-element sets; 'the integer $1 \bmod (n)$ ' that captures what is common within the equivalence class $\{\ldots,-2 n+1,-n+1,1, n+1,2 n+1, \ldots\}$ of integers; 'the circle' or 'the equilateral triangle'-and so forth 3

The notion of an entity that is partly indefinite and partly definite might be intuitively clarified by considering the difference between the two ways that police get a picture of a suspect, a mugbook and a police artist using a sketchpad. The mugbook is a set of definite images akin to the classical physics notion of reality. But a police sketch artist starts with an indefinite face on the sketchpad and then builds up more definiteness-which is akin to the notion of an indefinite quantum reality that is made more definite by a series of (compatible) measurements.

\subsection{The general notion of \#2 abstraction}

The type 2 abstraction is usually applied in mathematics to the elements of an equivalence class but we can apply the idea to any arbitrary (nonempty) subset $S \subseteq U$ to arrive at the idea of an abstract entity $u_{S}$ that is definite on what is common to the elements of $S$ and indefinite on where they differ. In terms of the abstraction principle: $u_{S}(u)=u_{S}\left(u^{\prime}\right)$ iff $u, u^{\prime} \in S$, for all $u, u^{\prime} \in U$. Intuitively, we might use the crutch of thinking of $u_{S}$ as resulting from blobbing, blurring, or smearing together the elements of $S$ to obtain $u_{S}$-so the only definite characteristics left in $u_{S}$ are the common properties and the properties where the elements of $S$ differ are blurred out as indefinite. But we need a more exact way to specify the difference between $S$ and $u_{S}$.

The notion of the incidence matrix $I(R)$ of a binary relation $R \subseteq U \times U$ on $U$ supplies the right mathematical notion to distinguish $S$ and $u_{S}$; it is the $n \times n$ matrix with rows and columns corresponding to the elements $u_{1}, \ldots, u_{n} \in U$ such that:

$$
I(R)_{i j}=\left\{\begin{array}{l}
1 \text { if }\left(u_{i}, u_{j}\right) \in R \\
0 \text { otherwise }
\end{array}\right.
$$

Then the set $S \subseteq U$ of distinct elements $u_{i} \in S$ could be represented by the incidence matrix $I(\Delta S)$ of the binary relation $\Delta S=\left\{\left(u_{i}, u_{i}\right): u_{i} \in S\right\}$ whose only non-zero elements are the diagonal elements of 1 corresponding to the $u_{i} \in S$. Then the "blobbed-out" or "blurred" version $u_{S}$ abstracted from $S$ would be represented by the incidence matrix $I(S \times S)$ with the entries $I(S \times S)_{i j}=1$ if

\footnotetext{
${ }^{3}$ Category theory helped to motivate homotopy type theory for good reason. Category theory has no notion of identity between objects, only isomorphism as 'equivalence' between objects. Therefore category theory can be seen as a theory of abstract \#2 objects (i.e., the \#2 abstract of an isomorphism class), e.g., abstract sets, groups, spaces, etc.
} 
$u_{i}, u_{j} \in S$ and 0 otherwise. The non-zero off-diagonal elements $I(S \times S)_{i j}=1$ for $i \neq j$ indicated that $u_{i}, u_{j} \in S$ are blobbed or blurred together or 'cohere' together in the entity $u_{S}$.

\subsection{From incidence to density matrices}

If the incidence matrices $I(\Delta S)$ and $I(S \times S)$ are normalized by dividing through by their trace $|S|$, then we obtain two density matrices denoted:

$$
\rho(\Delta S)=\frac{1}{\operatorname{tr}[I(\Delta S)]} I(\Delta S) \text { and } \rho(S)=\frac{1}{\operatorname{tr}[I(S \times S)]} I(S \times S) .
$$

If we assign the equal probabilities to the elements of $S: \operatorname{Pr}\left(u_{i}\right)=p_{i}=\frac{1}{|S|}$ if $u_{i} \in S$ and 0 otherwise, then the diagonal elements of $\rho(\Delta S)$ and $\rho(S)$ are the probabilities of drawing the corresponding element from $U$.

This incidence matrix approach to density matrices can be generalized by starting with any set of point probabilities $\operatorname{Pr}\left(u_{i}\right)=p_{i}$ for $u_{i} \in U$. Then the subset $S$ could be represented as a normalized column vector $|S\rangle$ which $i^{\text {th }}$ entry is $\sqrt{\frac{p_{i}}{\operatorname{Pr}(S)}}$ if $u_{i} \in S$ and 0 otherwise where $\operatorname{Pr}(S)=\sum_{u_{i} \in S} p_{i}$. Then the density matrix $\rho(S)$ would be constructed as the (outer) product of the column vector $|S\rangle$ times its transpose denoted $\langle S|=| S\rangle^{t}$ :

$$
\rho(S)_{i j}=(|S\rangle\langle S|)_{i j}=\frac{1}{\operatorname{Pr}(S)} \sqrt{p_{i} p_{j}} \text { if } u_{i}, u_{j} \in S \text { and } 0 \text { otherwise. }
$$

Then a density matrix $\rho(\pi)$ can be associated with a partition $\pi=\left\{B_{1}, \ldots, B_{m}\right\}$ on $U$ with the point probabilities $p=\left\{p_{1}, \ldots, p_{n}\right\}$ by taking the probability weighted sum of the density matrices for the blocks $B_{j}$ of $\pi$ :

$$
\rho(\pi)=\sum_{j=1}^{m} \operatorname{Pr}\left(B_{j}\right) \rho\left(B_{j}\right) .
$$

Then a non-zero off-diagonal entry $\rho(\pi)_{i i^{\prime}}=\sqrt{p_{i} p_{i^{\prime}}}$ means that $u_{i}$ and $u_{i^{\prime}}$ cohere together in some block $B_{j}$ and that $\left(u_{i}, u_{i^{\prime}}\right) \in \operatorname{indit}(\pi)$ is an indistinction of the partition $\pi$. Those non-zero offdiagonal entries $\rho(\pi)_{i i^{\prime}}=\sqrt{p_{i} p_{i^{\prime}}}$ can be thought of as an "amplitude" for $u_{i}$ and $u_{i^{\prime}}$ to cohere together since the square $p_{i} p_{i^{\prime}}$ is the probability that the ordered pair indit $\left(u_{i}, u_{i^{\prime}}\right)$ will be drawn (in that order) in two independent draws from the sample space $U$.

There is zero coherence amplitude in $\rho(\pi)$ for elements $u_{i}$ and $u_{i^{\prime}}$ in different blocks of $\pi$, i.e., for $\left(u_{i}, u_{i^{\prime}}\right) \in \operatorname{dit}(\pi)$. The most decoherent partition (with no coherence amplitudes) is the discrete partition $\mathbf{1}_{U}=\left\{\left\{u_{i}\right\}\right\}_{i=1, \ldots, n}$ with all the blocks are singletons so no elements of $U$ are blobbed or blurred together. Then $\rho\left(\mathbf{1}_{U}\right)$ is diagonal matrix with diagonal entries $\rho\left(\mathbf{1}_{U}\right)_{i i}=p_{i}$.

\subsection{Density matrices in quantum mechanics}

The transition to $\mathrm{QM}$ is rather clear. The elements $u_{i} \in U$ generalize to the vectors $\left|u_{i}\right\rangle$ in an orthonormal $(\mathrm{ON})$ basis $\mathcal{U}=\left\{\left|u_{i}\right\rangle\right\}_{i=1, \ldots, n}$ for an $n$-dimensional Hilbert space $V$. A superposition state $|\psi\rangle \in V$ can be represented as a superposition of vectors in the ON basis:

$$
|\psi\rangle=\sum_{i=1}^{n}\left\langle u_{i} \mid \psi\right\rangle\left|u_{i}\right\rangle=\sum_{i} \alpha_{i}\left|u_{i}\right\rangle
$$

where $\alpha_{i}=\left\langle u_{i} \mid \psi\right\rangle$. The first classical approximation to a superposition state was the blobbed-out or blurred version $u_{S}$ of a subset $S$ which was definite on the attributes common to the elements of $S$ and indefinite concerning the properties that differ between the elements of $S$. This blurred version $u_{S}$ of $S$ could be represented by the incidence matrix $I(S \times S)$ where two elements $u_{i}$ and $u_{j}$ cohered or were blurred together iff they were both in $S$. The normalized incidence matrix $\frac{1}{\operatorname{tr}[I(S \times S)]} I(S \times S)$ was a density matrix that could be further refined by introducing different point probabilities $p=\left\{p_{1}, \ldots, p_{n}\right\}$. Then we have the density matrix $\rho(S)$ whose non-zero off-diagonal 
entries $\frac{1}{\operatorname{Pr}(S)} \sqrt{p_{i} p_{i^{\prime}}}$ give the amplitude for $u_{i}$ to cohere or blur together with $u_{i^{\prime}}$ in $S . \rho(S)$ is our final classical representation of the \#2 abstraction from $S$ : from $u_{S}$ to $I(S \times S)$ to $\frac{1}{\operatorname{tr}[I(S \times S)]} I(S \times S)$ to $\rho(S)$.

The quantum version of $\rho(S)$ is $\rho(\psi)=|\psi\rangle\langle\psi|$ with the entries $\rho(\psi)_{i j}=\alpha_{i} \alpha_{j}^{*}$ (where $\alpha_{j}^{*}$ is the complex conjugate of $\alpha_{j}=\left\langle u_{j} \mid \psi\right\rangle$ ). Then the non-zero off-diagonal elements $\alpha_{i} \alpha_{j}^{*}$ for $i \neq j$ give the amplitude for $\left|u_{j}\right\rangle$ to cohere or blur together with $\left|u_{j}\right\rangle$ in the superposition $|\psi\rangle$-the indistinction amplitude, and those elements are usually called "coherences" [8, p. 302]. This blurring together of elements in the classical \#2 abstraction is a key characteristic in the quantum case.

[The] off-diagonal terms of a density matrix...are often called quantum coherences because they are responsible for the interference effects typical of quantum mechanics that are absent in classical dynamics. [2, p. 177]

It might be useful to connect this notion of a superposition state as a partly definite and indefinite entity. i.e., that is definite only on the properties common to the superposed states and indefinite otherwise, to common examples such as the double-slit experiment or the Mach-Zehnder interferometer. In the double-slit experiment, consider the superposition state $|\psi\rangle=\frac{1}{\sqrt{2}}(\mid$ slit 1$\rangle+\mid$ slit 2$\left.\rangle\right)$. This is commonly described as the state of the particle as "going through two slits at the same time" [1, p. 94]. But that assumes that there is a definite particle that is going through each slit. But the objectively indefinite interpretation of QM would interpret the superposition $|\psi\rangle$ as the blurred-together state of being indefinite as to which slot the particle goes through-and only being definite on going through the slits. Abner Shimony found one of Yogi Berra's malapropisms to be quite appropriate: "If you come to a fork in the road, take it." [28, p. 5] We do not have a "clear and distinct idea' how to imagine such an indefinite state-although many find the crutch of a definite wave hitting both slits as being helpful (but misleading) imagery.

In the case of the Mach-Zehnder interferometer, the superposition state $|\phi\rangle=\frac{1}{\sqrt{2}}(|\operatorname{arm} 1\rangle+|\operatorname{arm} 2\rangle)$ (after the first beam-splitter) is often described as the photon going through both arms. But on the OI interpretation, it would be more accurate to say that the photon is in the state of being indefinite (i.e., blurred) between the two arms but is definitely going through the arms of the apparatus 4

The point might be illustrated using our mugbook-sketchpad analogy. Suppose a witness has found two pictures of different people in the mugbook that she thinks equally depict the suspect. That is analogous to the description of the particle as definitely going through both slits or both arms of apparatus. But the OI interpretation would take the proper analogy as being a partial sketch of the suspect that is partly definite (e.g., on the characteristics common to the two mugshots) and partly indefinite (e.g., on where the two mugshots differ). To extend the analogy to the mathematics, such a partial sketch could be represented as the superposition: $|\varphi\rangle=\frac{1}{\sqrt{2}}(\mid$ mugshot 1$\rangle+\mid$ mugshot 2$\left.\rangle\right)$.

\section{Interpreting the inner product}

\subsection{The classical case}

Classically, we might take the norm of a subset $S \subseteq U$ as $\|S\|=\sqrt{|S|}$, the square root of its cardinality. The amplitude of the overlap between sets $S, T \subseteq U$ is $\|S \cap T\|=\sqrt{|S \cap T|}$ so the square of that overlap amplitude is the cardinality $|S \cap T|$. In terms of our leifmotif of distinction and indistinction, $\|S \cap T\|$ measures the amplitude of indistinction between $S$ and $T$. The maximum value is when they are fully indistinct, $\|S \cap T\|=\|S \cap S\|=\|T \cap T\|$, and the minimum indistinction amplitude $\|S \cap T\|=0$ means they have no overlap and have no indistinctness, i.e., are fully distinct. If we compare a random drawing from $S$ to a random drawing from $T$, then we could always distinguish between the drawings no matter what the outcome iff $\|S \cap T\|=0$.

\footnotetext{
${ }^{4}$ For more on these and other apparatuses in the context of delayed-choice experiments, see 13
} 
Given the set $U=\left\{u_{1}, \ldots, u_{n}\right\}$, the coefficients $\left\|\left\{u_{i}\right\} \cap S\right\|$ represent the amplitude of $\left\{u_{i}\right\}$ 's indistinctness with $S$, and its square $\left\|\left\{u_{i}\right\} \cap S\right\|^{2}=\left|\left\{u_{i}\right\} \cap S\right|$ represents the proportion of $S$ that is $\left\{u_{i}\right\}$. The non-zero proportions $\left\|\left\{u_{i}\right\} \cap S\right\|^{2}=\left|\left\{u_{i}\right\} \cap S\right|$ add up to equal $\|S\|^{2}=|S|$. Taking the uniform probability distribution on $U$, the probability $\operatorname{Pr}\left(u_{i} \mid S\right)$ of drawing a particular element $u_{i} \in S$ is the normalized proportion $\frac{\left\|\left\{u_{i}\right\} \cap S\right\|^{2}}{\|S\|^{2}}=\frac{\left|\left\{u_{i}\right\} \cap S\right|}{|S|}=\frac{1}{|S|}$.

\subsection{The quantum case}

The quantum version of the overlap amplitude between two states $|\psi\rangle$ and $|\phi\rangle$ in $V$ is their inner product $\langle\phi \mid \psi\rangle$ which can be interpreted as the amplitude of their indistinctness. They are maximally indistinct when $\langle\phi \mid \psi\rangle=\langle\phi \mid \phi\rangle=\langle\psi \mid \psi\rangle$ and have no indistinctness, i.e., are fully distinct, when $\langle\phi \mid \psi\rangle=0$. When comparing the measurement of $|\psi\rangle$ to the measurement of $|\phi\rangle$ (using some ON measurement basis $\mathcal{U}=\left\{\left|u_{i}\right\rangle\right\}_{i=1, \ldots, n}$ ), they are fully distinguishable regardless of the outcome iff $\langle\phi \mid \psi\rangle=0$.

Given an $\mathrm{ON}$ basis $\mathcal{U}=\left\{\left|u_{i}\right\rangle\right\}_{i=1, \ldots, n}$, the coefficients $\left\langle u_{i} \mid \psi\right\rangle=\alpha_{i}$ represent the amplitude of $\left|u_{i}\right\rangle$ 's indistinctness with $|\psi\rangle$, and its absolute square $\alpha_{i} \alpha_{i}^{*}=\left\|\left\langle u_{i} \mid \psi\right\rangle\right\|^{2}$ represents the proportion of $|\psi\rangle$ that is $\left|u_{i}\right\rangle$ 5 The non-zero proportions $\left\|\left\langle u_{i} \mid \psi\right\rangle\right\|^{2}$ add up to equal $\|\langle\psi \mid \psi\rangle\|^{2}$. Taking the uniform probability distribution on the interval $\left[0,\|\langle\psi \mid \psi\rangle\|^{2}\right]$, the probability $p_{i}$ of a point falling in a segment of length $\left\|\left\langle u_{i} \mid \psi\right\rangle\right\|^{2}$ is just that normalized length of the segment $p_{i}=\frac{\left\|\left\langle u_{i} \mid \psi\right\rangle\right\|^{2}}{\|\langle\psi \mid \psi\rangle\|^{2}}$, which is also the probability of getting the outcome $\left|u_{i}\right\rangle$ when measuring $|\psi\rangle$ using $\left\{\left|u_{i}\right\rangle\right\}_{i=1, \ldots, n}$ as the measurement basis, i.e., the Born rule.

\section{$5 \quad$ Numerical attributes and measurement}

\subsection{The classical case}

Given a universe set $U=\left\{u_{1}, \ldots, u_{n}\right\}$ with point probabilities $p_{1}, \ldots, p_{n}$, a real-value numerical attribute on $U$ is a function $f: U \rightarrow \mathbb{R}$. The numerical values $\left\{r_{1}, \ldots, r_{m}\right\}$ in the image of $f$ define a partition $f^{-1}=\left\{B_{1}, \ldots, B_{m}\right\}$ on $U$ by taking $B_{j}=f^{-1}\left(r_{j}\right)$ for $j=1, \ldots, m$.

The blobbed, blurred, or smeared \#2 abstraction version of a nonempty subset $S \subseteq U$ is represented by the density matrix $\rho(S)$, which might be called a pure density matrix since $\rho(S)^{2}=$ $\rho(S)$ and thus $\operatorname{tr}\left[\rho(S)^{2}\right]=\operatorname{tr}[\rho(S)]=1$. Intuitively, this 'superposition' version of $S$ is definite only on the properties common to all the elements of $S$ and is otherwise indefinite. But the blurredtogether elements of superposition $S$ might be distinguished by classifying them according to some numerical attribute $f$. Since the superposition version of $S$ is represented by the density matrix $\rho(S)$, this classification operation might represented by an operation on the density matrix $\rho(S)$ to obtain an $f$-classified density matrix $\hat{\rho}(S)$. The non-zero off-diagonal elements $\rho(S)_{i i^{\prime}}=\frac{1}{\operatorname{Pr}(S)} \sqrt{p_{i} p_{i^{\prime}}}$ in $\rho(S)$ give the amplitude for $u_{i}$ to be indistinct with $u_{i^{\prime}}$ in the superposition version of $S$. The transformation $\rho(S) \rightsquigarrow \hat{\rho}(S)$ is quite simple; if $f$ distinguishes $u_{i}$ and $u_{i^{\prime}}$, i.e., if $\left(u_{i}, u_{i^{\prime}}\right)$ is a distinction of the partition $f^{-1}$, then and only then is the indistinction amplitude set to 0 . If $u_{i}$ and $u_{i^{\prime}}$ are not distinguished by $f$, i.e., $u_{i}$ and $u_{i^{\prime}}$ are not only both in $S$ but are both in some block of $f^{-1}=\left\{f^{-1}\left(r_{1}\right), \ldots, f^{-1}\left(r_{m}\right)\right\}$, then the indistinction amplitude $\frac{1}{\operatorname{Pr}(S)} \sqrt{p_{i} p_{i^{\prime}}}$ remains the same as before. And since no element $u_{i}$ can ever be distinguished from itself by any numerical attribute, the diagonal elements remain the same. These changes determine the $f$-classified density matrix $\hat{\rho}(S)$. Intuitively, the blurred or superposition version of $S$ represented by $\rho(S)$ has a definite attribute value only if that value is common to all the $u_{i} \in S$, i.e., for some $j, S \subseteq f^{-1}\left(r_{j}\right)=B_{j}$, and

\footnotetext{
${ }^{5}$ We use the notation $\|\alpha\|=\sqrt{\alpha \alpha^{*}}$ for the norm of a complex number $\alpha$ to avoid notational conflict with the cardinality $|S|$ of a subset $S$.
} 
then $\rho(S)=\hat{\rho}(S)$. Otherwise the elements of the set $S$ do not share any $f$-value so $\hat{\rho}(S)^{2} \neq \hat{\rho}(S)$, $\operatorname{tr}\left[\hat{\rho}(S)^{2}\right]<1$, and $\hat{\rho}(S)$ might be called a mixed density matrix.

The transformation of matrices $\rho(S) \rightsquigarrow \hat{\rho}(S)$ can also be specified entirely using matrix operations. Let $P_{B_{j}}$ be the diagonal $n \times n$ matrix with diagonal element $\left(P_{B_{j}}\right)_{i i}$ equal to 1 if $u_{i} \in B_{j}$ and 0 otherwise so it is a projection matrix $P_{B_{j}}^{2}=P_{B_{j}}$. Then pre- and post-multiplying $\rho(S)$ by the projections $P_{B_{j}}$ for $B_{j} \in f^{-1}$ and summing has the effect of zeroing out all the indistinction amplitudes $\frac{1}{\operatorname{Pr}(S)} \sqrt{p_{i} p_{i^{\prime}}}$ where $u_{i}$ and $u_{i^{\prime}}$ are distinguished by $f$. In anticipation of the quantum case, this operation on $\rho(S)$ to obtain the $f$-classified $\hat{\rho}(S)$ will be called the classical Lüders mixture operation:

$$
\hat{\rho}(S)=\sum_{j=1}^{m} P_{B_{j}} \rho(S) P_{B_{j}} .
$$

If $S=U$, the universe set, then $\rho(S)=\rho\left(\mathbf{0}_{U}\right)$, the density matrix representation of the indiscrete partition $\mathbf{0}_{U}=\{U\}$. The $j$ oin of two partitions $\pi=\left\{B_{j}\right\}_{j=1}^{m}$ and $\sigma=\left\{C_{k}\right\}_{k=1}^{m^{\prime}}$, is the partition $\pi \vee \sigma$ whose blocks are all the non-empty intersections $B_{j} \cap C_{k} \neq \emptyset$. The join operation combines all the distinctions made by the two partitions, i.e., $\operatorname{dit}(\pi \vee \sigma)=\operatorname{dit}(\pi) \cup \operatorname{dit}(\sigma)$. The result of classifying $\rho\left(\mathbf{0}_{U}\right)$ by $\pi=f^{-1}$ is the density matrix of the join $\mathbf{0}_{U} \vee \pi=\pi$, i.e., $\hat{\rho}\left(\mathbf{0}_{U}\right)=\rho(\pi)$. Further classifications by other partitions on $U$ will add to the join and thus introduce more and more distinctions until obtaining the maximally distinguished discrete partition $\mathbf{1}_{U}=\left\{\left\{u_{i}\right\}\right\}_{i=1}^{n}$. A set of partitions $\{\pi, \sigma, \ldots\}$ such that $\pi \vee \sigma \vee \ldots=\mathbf{1}_{U}$ might be called a complete set of partitions on $U$. The density matrix $\rho\left(\mathbf{1}_{U}\right)$ is the diagonal matrix $\rho(\Delta U)$ with the probabilities $p_{i}$ 's as the diagonal entries where all the blurring effects or indistinctions between the elements of the superposition version of $U$ have been eliminated.

\subsection{The quantum case}

Given an orthonormal basis $\mathcal{U}=\left\{\left|u_{i}\right\rangle\right\}_{i=1, \ldots, n}$ for the $n$-dimensional Hilbert space $V$, a real-valued numerical attribute is a function $f: \mathcal{U} \rightarrow \mathbb{R}$ with a set of image values $\left\{\lambda_{1}, \ldots, \lambda_{m}\right\}$. Extending the $f$-assignment $\left|u_{i}\right\rangle \longmapsto \lambda_{i}\left|u_{i}\right\rangle$ linearly to the whole space $V$ defines a linear operator $F: V \rightarrow V$ with eigenvectors $\mathcal{U}=\left\{\left|u_{i}\right\rangle\right\}_{i=1, \ldots, n}$ and real eigenvalues $\lambda_{1}, \ldots, \lambda_{m}$, so $F$ is a Hermitian operator, i.e., an observable. Conversely, each Hermitian operator $F: V \rightarrow V$ has an ON basis $\mathcal{U}=\left\{\left|u_{i}\right\rangle\right\}_{i=1, \ldots, n}$ of eigenvectors with eigenvalues $\lambda_{1}, \ldots, \lambda_{m}$ so that assigning each eigenvector its eigenvalue gives the eigenvalue function which is a numerical attribute $f: \mathcal{U} \rightarrow \mathbb{R}$.

Given a normalized superposition state $|\psi\rangle$, its resolution in terms of an ON basis $\mathcal{U}$ of eigenvectors of a Hermitian operator $F$ gives $|\psi\rangle=\sum_{i=1}^{n}\left\langle u_{i} \mid \psi\right\rangle\left|u_{i}\right\rangle=\sum_{i} \alpha_{i}\left|u_{i}\right\rangle$. The density matrix $\rho(\psi)=|\psi\rangle\langle\psi|$ represented in the $\mathcal{U}$-basis has the elements $\rho(\psi)_{i i^{\prime}}=\alpha_{i} \alpha_{i^{\prime}}^{*}$ and is a pure state density matrix where $\rho(\psi)^{2}=\rho(\psi)$ and $\operatorname{tr}\left[\rho(\psi)^{2}\right]=1$. Let $f: \mathcal{U} \rightarrow \mathbb{R}$ be the eigenvalue function assigning to each eigenvector $\left|u_{i}\right\rangle$ its eigenvalue where $\lambda_{1}, \ldots, \lambda_{m}$ are the eigenvalues of $F$. The inverse images $f^{-1}\left(\lambda_{j}\right)$ define a set partition $f^{-1}$ on the set $\mathcal{U}$ where each block $B_{j}=f^{-1}\left(\lambda_{j}\right)$ generates the eigenspace $\left[B_{j}\right] \subseteq V$ associated with the eigenvalue $\lambda_{j}$ for $\left.j=1, \ldots, m\right]$ The observable $F$, or equivalently the eigenvalue function $f: \mathcal{U} \rightarrow \mathbb{R}$, can be used to distinguish or classify the states $\left|u_{i}\right\rangle$ that are blurred together in the superposition state $|\psi\rangle$ with the indistinction amplitudes or coherences $\alpha_{i} \alpha_{i^{\prime}}^{*}$ between the states $\left|u_{i}\right\rangle$ and $\left|u_{i^{\prime}}\right\rangle$. This operation of distinguishing by classifying, usually called "(projective) measurement", has the same effect on the density matrix $\rho(\psi)$ (represented in the measurement basis $\mathcal{U}$ ) of zeroing out (or decohering) the indistinction amplitudes $\rho(\psi)_{i i^{\prime}}=\alpha_{i} \alpha_{i^{\prime}}^{*}$

\footnotetext{
${ }^{6}$ The eigenspaces $\left[B_{j}\right]$ form a direct-sum decomposition of $V$. A direct-sum decomposition of a vector space can be considered the vector-space version of a partition on a set. Since a set-partition (or quotient set) is categorytheoretically dual to a subset, a direct-sum decomposition of a vector space is similarly dual to a subspace. And just as the Boolean logic of subsets has the dual logic of partitions, so the usual notion of the quantum logic of (closed) subspaces of a Hilbert space [4] will have a dual form in the quantum logic of direct-sum decompositions [17.
} 
when and only when $\left|u_{i}\right\rangle$ and $\left|u_{i^{\prime}}\right\rangle$ are distinguished by $f$, i.e., have different eigenvalues. Using the same projection matrices $P_{B_{j}}$ where $B_{j}=f^{-1}\left(\lambda_{j}\right)$ as in the classical case, the post-classification or post-measurement density matrix $\hat{\rho}(\psi)$ is obtained by the quantum Lüders mixture operation [2, p. 279]:

$$
\hat{\rho}(\psi)=\sum_{j=1}^{m} P_{B_{j}} \rho(\psi) P_{B_{j}} .
$$

If $G: V \rightarrow V$ is another Hermitian operator on $V$ that commutes with $F$, then we can take the ON basis $\mathcal{U}$ as a basis of simultaneous eigenvectors of both $F$ and $G$. If $g: \mathcal{U} \rightarrow \mathbb{R}$ is the eigenvalue function of $G$ with eigenvalues $\mu_{1}, \ldots, \mu_{m^{\prime}}$, then $g^{-1}$ gives a set partition on $\mathcal{U}$ and the join $f^{-1} \vee g^{-1}$ is a more refined partition on $\mathcal{U}$ where each block $f^{-1}\left(\lambda_{j}\right) \cap g^{-1}\left(\mu_{k}\right)$ can be characterized by the ordered pair $\left(\lambda_{j}, \mu_{k}\right)$ of eigenvalues. A set of commuting operators $F, G, \ldots$ is called a complete set of commuting operators (CSCO) if all the blocks in the join $f^{-1} \vee g^{-1} \vee \ldots$ are singletons so each basis simultaneous eigenvector in $\mathcal{U}$ can be characterized by the sequence of eigenvalues $\left(\lambda_{j}, \mu_{k}, \ldots\right)$. If all the compatible measurements by the observables in a CSCO have been carried out, then the result is the completely decohered diagonal density matrix with all the off-diagonal coherence amplitudes eliminated.

Definiteness in QM is achieved when a state such as $\left|u_{i}\right\rangle$ has a specific eigenvalue $f\left(\left|u_{i}\right\rangle\right)=\lambda_{j}$. Intuitively, in a blobbed, blurred, or smeared state such as a superposition $|\psi\rangle$, it is definite only on the attributes that are common to all the $\left|u_{i}\right\rangle$ ' $i n$ ' $|\psi\rangle$ (in the sense that $\left\langle u_{i} \mid \psi\right\rangle \neq 0$ ), and indefinite otherwise. In more precise terms, a superposition state $|\psi\rangle$ has the definite $F$-observable value of $\lambda_{j}$ if and only if all the $\left|u_{i}\right\rangle$ in the superposition $|\psi\rangle$ also have that same value $\lambda_{j}$-in which case $\hat{\rho}(\psi)=\rho(\psi)$ and $|\psi\rangle \in\left[B_{j}\right]$, i.e., $|\psi\rangle$ is one of the eigenvectors for $\lambda_{j}$. Otherwise, the $\left|u_{i}\right\rangle$ in $|\psi\rangle$ have no $F$-value in common so $\hat{\rho}(\psi) \neq \rho(\psi), \operatorname{tr}\left[\hat{\rho}(\psi)^{2}\right]<1$, and $\hat{\rho}(\psi)$ is the density matrix of a mixture.

A more non-trivial example of a partly definite and partly indefinite state is the definite correlation obtained in an entangled superposition. Suppose an observable $A$ can have two eigenstates $\left|a_{1}\right\rangle$ and $\left|a_{2}\right\rangle$ in a Hilbert state $H$ and an observable $B$ has two eigenstates $\left|b_{1}\right\rangle$ and $\left|b_{2}\right\rangle$ in another Hilbert space $H^{\prime}$. Then in the tensor product $H \otimes H^{\prime}$, we have the definite states $|s\rangle=\left|a_{1}\right\rangle \otimes\left|b_{1}\right\rangle$ and $\left|s^{\prime}\right\rangle=\left|a_{2}\right\rangle \otimes\left|b_{2}\right\rangle$, but the entangled superposition state $F=\frac{1}{\sqrt{2}}\left(\left|a_{1}\right\rangle \otimes\left|b_{1}\right\rangle+\left|a_{2}\right\rangle \otimes\left|b_{2}\right\rangle\right)$ is not definitely in either state.

When the composite system is in the state $F$, however, neither $A$ nor $B$ has a definite value, but there is a definite correlation of $A$ and $B: A$ and $B$ are actualized jointly either as $\left(a_{1}, b_{1}\right)$ or as $\left(a_{2}, b_{2}\right)$. The composite system has a definite property, which can loosely be called "sameness of the indices of the possible values of A and B," not inferrable from the entire specification of $s$ by itself and the entire specification of $s^{\prime}$ by itself. [28, p. 7]

\section{Logical information theory at the classical and quantum level}

\subsection{The classical case}

The strategy of elucidating the objective indefiniteness interpretation of QM is to use the notions distinction and indistinction, distinguishability and indistinguishability, first in the classical case, where they are more easily understood, and then to recapitulate them in the quantum case. The notion of logical entropy at the classical and quantum level captures quantitatively the creation of distinctions from indistinctions in classification and measurement.

Given a set partition $\pi=\left\{B_{1}, \ldots, B_{m}\right\}$ on a set $U=\left\{u_{1}, \ldots, u_{n}\right\}$, the set of distinctions or dits of $\pi$ is the set $\operatorname{dit}(\pi) \subseteq U \times U$ of all ordered pairs $\left(u_{i}, u_{i^{\prime}}\right)$ with $u_{i}$ and $u_{i^{\prime}}$ in different blocks of $\pi$. If all 
the points of $U$ are equiprobable, i.e., $p_{i}=\frac{1}{|U|}$, then the logical entropy of $\pi$, denoted $h(\pi)$, is the normalized count of the distinctions of $\pi$, i.e., $h(\pi)=\frac{|\operatorname{dit}(\pi)|}{|U \times U|}$. With $\operatorname{Pr}\left(B_{j}\right)=\sum\left\{p_{i}: u_{i} \in B_{j}\right\}$ and the complementary equivalence relation indit $(\pi)=U \times U-\operatorname{dit}(\pi)=\cup_{j=1}^{m} B_{j} \times B_{j}$, we can express the logical entropy as:

$$
h(\pi)=\frac{|\operatorname{dit}(\pi)|}{|U \times U|}=\frac{\left|U \times U-\cup_{j} B_{j} \times B_{j}\right|}{|U \times U|}=1-\sum_{j} \frac{\left|B_{j} \times B_{j}\right|}{|U \times U|}=1-\sum_{j}\left(\frac{\left|B_{j}\right|}{|U|}\right)^{2}=1-\sum_{j=1}^{m} \operatorname{Pr}\left(B_{j}\right)^{2} .
$$

When $U$ has point probabilities $p_{1}, \ldots, p_{n}$, then the natural definition is: $h(\pi)=1-\sum_{j=1}^{m} \operatorname{Pr}\left(B_{j}\right)^{2}$. The logical entropy of a partition $h(\pi)$ has the simple interpretation: in two independent draws (i.e., with replacement) from $U, h(\pi)$ is the probability of drawing a distinction of $\pi$-and $\sum_{j} \operatorname{Pr}\left(B_{j}\right)^{2}$ is the complementary probability of drawing an indistinction of $\pi$.

The two extreme partitions are the indiscrete partition (or 'blob') $\mathbf{0}_{U}=\{U\}$ which makes no distinctions so $h\left(\mathbf{0}_{U}\right)=0$, and the discrete partition $\mathbf{1}_{U}=\left\{\left\{u_{i}\right\}\right\}_{i=1, \ldots, n}$ which distinguishes all the elements of $U$ so $h\left(\mathbf{1}_{U}\right)=1-\sum_{i=1}^{n} p_{i}^{2}$. The maximum logical entropy occurs in the equiprobable case of $p_{i}=\frac{1}{|U|}=\frac{1}{n}$ when $h\left(\mathbf{1}_{U}\right)=1-\frac{1}{n}$ which is the two-draw probability of drawing distinct elements of $U$.

The definitions are easily reformulated in terms of the density matrix representation of the partition as: $\rho(\pi)=\sum_{j=1}^{m} \operatorname{Pr}\left(B_{j}\right) \rho\left(B_{j}\right)$. Then the equivalent definition of the logical entropy $h(\pi)$ of $\pi$ is:

$$
h(\rho(\pi))=1-\operatorname{tr}\left[\rho(\pi)^{2}\right] .
$$

A pure density matrix $\rho(S)$ representing the superposition-version of $S \subseteq U$ has $\rho(S)^{2}=\rho(S)$ and all density matrices have trace 1 so the logical entropy of pure density matrices is always zero: $h(\rho(S))=1-\operatorname{tr}\left[\rho(S)^{2}\right]=1-1=0$. We have seen that the classification or distinguishing of the blobbed-together elements of $S$ by a partition $\pi$ transforms the pure density matrix $\rho(S)$ into the mixed density matrix $\hat{\rho}(S)$ obtained by the classical Lüders mixture operation $\hat{\rho}(S)=\sum_{j=1}^{m} P_{B_{j}} \rho(S) P_{B_{j}}$. The classification zeros all the off-diagonal indistinction-amplitude terms $\frac{1}{\operatorname{Pr}(S)} \sqrt{p_{i} p_{i^{\prime}}}$ for $u_{i}, u_{i^{\prime}} \in S$ where $u_{i}$ and $u_{i^{\prime}}$ are in different blocks of $\pi$. Logical entropy captures these distinctions made by the classification of $\rho(S)$ by the partition $\pi$. The fundamental theorem relating logical entropy and classification is:

Theorem 1 The sum of the squares of all indistinction-amplitudes zeroed in the Lüders mixture operation taking $\rho(S)$ to $\hat{\rho}(S)$ is the logical entropy $h(\hat{\rho}(S))$. 14]

\subsection{The quantum case}

The quantum case is a straight-forward generalization of the classical case. The quantum logical entropy of any quantum state given by a density matrix $\rho$ is defined by:

$$
h(\rho)=1-\operatorname{tr}\left[\rho^{2}\right] .
$$

Let $\mathcal{U}=\left\{\left|u_{i}\right\rangle\right\}_{i=1, \ldots, n}$ again be an orthonormal basis of eigenvectors of a Hermitian operator $F$ : $V \rightarrow V$ with eigenvectors $\left\{\lambda_{j}\right\}_{j=1, \ldots, m}$. Let $|\psi\rangle=\sum_{i} \alpha_{i}\left|u_{i}\right\rangle$ be a normalized superposition state with $\rho(\psi)=|\psi\rangle\langle\psi|$ so that $h(\rho(\psi))=0$. The measurement of $|\psi\rangle$ by the observable $F$ transforms the pure state density operator $\rho(\psi)$ into the mixed state density operator given by the Lüders mixture operation: $\hat{\rho}(\psi)=\sum_{j=1}^{m} P_{j} \rho(\psi) P_{j}$ where $P_{j}$ is the projection to the eigenspace of the eigenvalue $\lambda_{j}$. Then the quantum logical entropy $h(\hat{\rho}(\psi))=1-\operatorname{tr}\left[\hat{\rho}(\psi)^{2}\right]$ has the simple interpretation of the being the probability in two independent $F$-measurements of $|\psi\rangle$ of getting different eigenvalues. 
Moreover, the fundamental theorem relating quantum logical entropy to (projective) measurement gives the detailed connection to the changes in the density matrix $\rho(\psi)$ when represented in the $U$-basis 7

Theorem 2 The sum of the absolute squares of all the indistinction-amplitudes or coherences $\alpha_{i} \alpha_{i^{\prime}}^{*}$ that are zeroed (i.e., decohered) in the Lüders mixture operation taking $\rho(\psi)$ to $\hat{\rho}(\psi)$ is the quantum logical entropy $h(\hat{\rho}(\psi))$. [16]

The notions of classical and quantum logical entropy give the respective measures of information based on the foundational idea of information-as-distinctions 8

\section{Quantum dynamics and measurement}

\subsection{Von Neumann's type 2 processes}

Von Neumann divided quantum processes into two fundamentally different types:

1. "the arbitrary changes by measurements," and

2. "the automatic changes which occur with passage of time." [32, p. 351]

The OI interpretation needs to 'make sense' out of these two different types of processes in terms of distinctions and indistinctions. Indeed, the difference is between:

1. processes that make distinctions, and

2. processes that preserve distinctions.

Taking \#2 first, The degree to which two quantum states are indistinct or distinct is given by the inner product $\langle\phi \mid \psi\rangle$, so a quantum process that does not change this amplitude of indistinction between states is mathematically described as a unitary transformation (i.e., a linear transformation that preserves inner products). The unitary evolution of superpositions, e.g., $|\psi(t)\rangle=U\left(t, t_{0}\right)\left|\psi\left(t_{0}\right)\right\rangle$, is a mathematical description of the propagation of waves. The connection between unitary transformations and the solutions to the Schrödinger "wave" equation is given by Stone's Theorem [30: there is a one-to-one correspondence between strongly continuous 1-parameter unitary groups $\left\{U\left(t, t_{0}\right)\right\}_{t \in \mathbb{R}}$ and Hermitian operators $H$ on the Hilbert space so that $U\left(t, t_{0}\right)=e^{i H t}$.

In simplest terms, a unitary transformation describes a rotation such as the rotation of a unit vector in the complex plane.
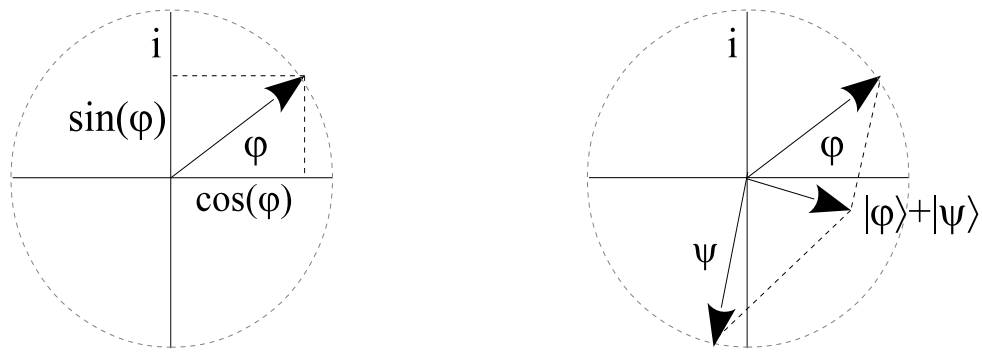

\footnotetext{
${ }^{7}$ Both quantum logical entropy and the Von Neumann entropy $S(\rho(\psi))=-\rho(\psi) \log (\rho(\psi))$ usually considered in QM have the value of 0 for pure states and increase under (projective) measurement. But there seems to be no similar relation between the Von Neumann entropy and the changes in the density matrix due to a measurement.

${ }^{8}$ The above classical cases, dealing with sets instead of vectors, could be made even closer to QM by using a vector space where each vector (represented in a basis set) is a set. That is the case for vector spaces over $\mathbb{Z}_{2}$. The above machinery from the classical cases formulated over $\mathbb{Z}_{2}^{n}$ gives a pedagogical (or "toy") model of QM-"quantum mechanics over sets." 15
} 
Figure 2: Rotating vector and addition of vectors

The rotating unit vector traces out the cosine and sine "wave" functions on the two axes, and the position of the arrow can be compactly described as a function of $\varphi$ using Euler's formula:

$$
e^{i \varphi}=\cos (\varphi)+i \sin (\varphi)
$$

Such complex exponentials and their superpositions are the "wave functions" of QM. The "wave functions" describe the evolution of particles in indefinite states in isolated systems where there are no distinction-creating interactions to change the degree of indistinctness between states, i.e., the context where Schrödinger's equation holds. Classically it has been assumed that the mathematics of waves must describe physical waves of some sort, and thus the puzzlement about the "wave functions" of QM having complex amplitudes (in $3 N$-dimensional space for systems of $N$ particles) and no corresponding physical waves.

But we have supplied another interpretation; wave mathematics is the mathematics of indefinitenesspreserving evolution, i.e., superposition represents indefiniteness and unitary evolution represents the indistinctness-preserving evolution of an isolated system. The same mathematics describes both types of evolution. Using the wave interpretation instead of the indefiniteness interpretation of the mathematics has been one of most historic wrong turns in the interpretation of QM-which has continued long after it was realized that the "wave function" could not describe actual physical waves. But humans have evolved so they can readily imagine the evolution of common macro-phenomena such as the propagation of waves, while indefinite states and their evolution present a much greater challenge to the imagination.

Richard Feynman's approach to QM shows how to develop the mathematics of QM without appeal to waves (although wave imagery may be used as a pedagogical crutch).

I want to emphasize that light comes in this form-particles. It is very important to know that light behaves like particles, especially for those of you who have gone to school, where you were probably told something about light behaving like waves. I'm telling you the way it does behave-like particles. [19, p. 15]

Indeed, Feynman takes note of cases where the wave theory falls short since:

the wave theory cannot explain how the detector makes equally loud clicks as the light gets dimmer. Quantum electrodynamics "resolves" this wave-particle duality by saying that light is made of particles (as Newton originally thought), but the price of this great advancement of science is a retreat by physics to the position of being able to calculate only the probability that a photon will hit a detector, without offering a good model of how it actually happens. [19, pp. 36-7]

The OI interpretation argues that what "actually happens" in "wave-like" behavior is the evolution of a particle that is indefinite between a number of undistinguished alternatives (a type 2 process), and thus the OI interpretation could be seen as attempting to give an ontology that underlies Feynman's mathematical approach to QM. For instance, in the double slit experiment, instead of saying "the electron sweeps from source to screen following all possible paths at once" [9, p. 32], it would be better to say that the electron was in a state of being indefinite between all the possible paths in going from source to screen. By developing the indefiniteness interpretation to the superposition of paths in the Feynman approach, one has a realistic non-wave interpretation of QM.

\subsection{Von Neumann's type 1 processes}

The \#1 type process is a process that does make distinctions. Richard Feynman has given perhaps the clearest characterization of the two types of processes in terms of distinctions and indistinctions. 
If you could, in principle, distinguish the alternative final states (even though you do not bother to do so), the total, final probability is obtained by calculating the probability for each state (not the amplitude) and then adding them together. If you cannot distinguish the final states even in principle, then the probability amplitudes must be summed before taking the absolute square to find the actual probability. [20, p. 3-16]

Feynman gives examples that do not involve any macroscopic measuring apparatus (neutrons scattering in crystals or collisions of alpha-particles) to avoid all the extraneous considerations (e.g., environmental dephasing) in the literature on measurement. For instance, Feynman considers the case where "all neutrons from the source having spin up and all the nuclei of the crystal having spin down" [20, p. 3-15] If a scattered neutron has spin down, then one of the atoms in the crystal must have spin up so the different paths through the crystal are distinguished. That is a type 1 process which makes distinctions between the paths so the amplitude of each path is (separately) squared to find its probability. If the alternatives cannot in principle be distinguished, then it is a type 2 process of unitary evolution of the indefinite superposition of the paths, so the path amplitudes are added before taking the absolute squares to determine the probability-which will then reflect the interference between the paths.

One can extract from Feynman's probability rules the basic distinguishability principle that separates type 2 unitary evolution from the type 1 state reduction or 'measurement.' Consider the unitary evolution of a particle in an indefinite state that is a superposition of various definite states. If the particle then undergoes an interaction where the outcomes of the superposed definite states can, in principle, be distinguished, then the states are distinguished and the particle emerges from the interaction in one of the definite states with the probability determined by the absolute square of its amplitude in the superposition (Born rule). In short, if an interaction has to make a difference between the superposed states in the final outcomes, then it does make a difference in that the indefinite superposition state is reduced to one of the definite states that were superposed.

Hermann Weyl likened a measurement to a particle having to pass through a "sieve or grating" [33. p. 259]. For an intuitive image, think of a "blob" of dough as the indefinite superposition of a set of polygonal shapes. The blob evolves as a blob until it hits a grating with holes corresponding to the superposed shapes so the blob then has to pass through one of the holes and thus gets 'ontologically classified' as one of the definite shapes. The grating distinguishes and classifies the shapes in the indefinite superposition.

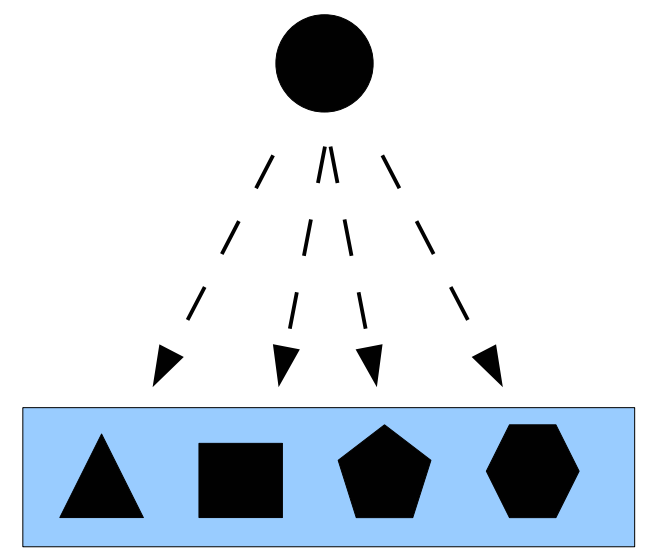

Figure 3: Measurement where a superposition of definite shapes has to take on one of the shapes.

Werner Heisenberg is usually presented as an advocate of the Copenhagen interpretation of QM. But in his mature philosophical reflections, e.g., 23, he used the imagery of "potentiality" and "actuality" which, as noted by Shimony, can be interpreted as "indefiniteness" and "definiteness" respectively. 
Heisenberg [23, p. 53] used the term "potentiality" to characterize a property which is objectively indefinite, whose value when actualized is a matter of objective chance, and which is assigned a definite probability by an algorithm presupposing a definite mathematical structure of states and properties. Potentiality is a modality that is somehow intermediate between actuality and mere logical possibility. That properties can have this modality, and that states of physical systems are characterized partially by the potentialities they determine and not just by the catalogue of properties to which they assign definite values, are profound discoveries about the world, rather than about human knowledge. It is fair to say, in view of my discussion above of metaphysics, that these statements about quantum mechanical potentiality are metaphysical propositions suggested by the formalism of quantum mechanics. These statements, together with the theses about potentiality, may collectively be called "the Literal Interpretation" of quantum mechanics. [28, p. 6]

Heisenberg's use of the notion of "potentiality" in contrast to actuality does not seem appropriate since the "potentialities" have very real effects on actuality (e.g., the quantum interference effects), so it would seem more appropriate to consider indefinite and definite actualities.

\section{Group theory and QM}

We have argued that reality at the quantum level is inherently indefinite which under certain circumstances becomes partly definite. The understanding of quantum reality in terms of indistinguishability is already well-known in the area of "identical" particles, e.g., [7], and that will not be recapitulated here. We have also emphasized the new light thrown on these questions by the new developments in the logic of partitions (= equivalence relations $=$ quotient sets) and in its quantitative development as logical information theory. There is another already well-known area of mathematics dealing the specification of equivalences (or symmetries), namely, group theory-so one would expect it to be highly applicable to QM. And it is.

An equivalence relation is a transitive, symmetric, and reflexive relation. A group operating on a set is a natural way to define an equivalence on the set (the partition of orbits) since a group operation is an associative operation that is closed under composition (transitivity), has inverses (symmetry), and includes the identity operation (reflexivity) [for more, see [6].

To briefly touch on a quantum example, we need to lift or generalize the set case of a group operating on a set, i.e., a set representation of the group operations, to complex vector space representations of a (symmetry) group. As noted above, a set partition generalizes to a direct-sum decomposition of a vector space. The set partition of orbits generalizes to the direct-sum decomposition of a complex vector space into irreducible subspaces. A representation restricted to an irreducible subspace is an irreducible representation. For a certain symmetry group of particle physics, "an elementary particle 'is' an irreducible unitary representation of the group." [29, p. 149] Thus our approach from partitions and equivalence relations comports with "the soundness of programs that ground particle properties in the irreducible representations of symmetry transformations..." [22, p. 171] (for more, see [18]).

\section{Concluding remark}

One way to succinctly describe the objective indefiniteness interpretation of QM is that the mathematics for the evolution of the quantum "wave function" is also the mathematics for the indistinctionpreserving evolution of indefinite (superposition) states. The so-called "wave-particle duality" is really the juxtaposition of a particle evolving with an indefinite position ("wave-like" behavior) with a particle having a definite position. The objective indefiniteness approach to interpreting QM thus 
provides an explanation for the appearance of the mathematics of waves (which implies interference as well as the quantized solutions to the "wave" equation that gave QM its name) when, in fact, there are no actual physical waves involved.

\section{References}

[1] Ananthaswamy, Anil. 2018. Through Two Doors At Once: The Elegant Experiment That Captures the Enigma of Our Quantum Reality. New York: Dutton.

[2] Auletta, Gennaro, Mauro Fortunato, and Giorgio Parisi. 2009. Quantum Mechanics. Cambridge UK: Cambridge University Press.

[3] Baues, Hans-Joachim. 1995. Homotopy Types. In Handbook of Algebraic Topology, edited by I. M. James, 1-72. Amsterdam: Elsevier Science.

[4] Birkhoff, Garrett, and John Von Neumann. 1936. "The Logic of Quantum Mechanics." Annals of Mathematics 37 (4): 823-43.

[5] Boole, George. 1854. An Investigation of the Laws of Thought on Which Are Founded the Mathematical Theories of Logic and Probabilities. Cambridge: Macmillan and Co.

[6] Brading, Katherine, and Elena Castellani. 2003. Symmetries in Physics: Philosophical Reflections. Cambridge UK: Cambridge University Press.

[7] Castellani, Elena, ed. 1998. Interpreting Bodies: Classical and Quantum Objects in Modern Physics. Princeton: Princeton University Press.

[8] Cohen-Tannoudji, Claude, Bernard Diu, and Franck Laloë. 2005. Quantum Mechanics: Volumes 1 and 2. New York: John Wiley \& Sons.

[9] Cox, Brian, and Jeff Forshaw. 2011. The Quantum Universe (and Why Anything That Can Happen, Does). Boston: DaCapo Press.

[10] Ellerman, David. 2009. "Counting Distinctions: On the Conceptual Foundations of Shannon's Information Theory." Synthese 168 (1 May): 119-49.

[11] Ellerman, David. 2010. "The Logic of Partitions: Introduction to the Dual of the Logic of Subsets." Review of Symbolic Logic 3 (2 June): 287-350.

[12] Ellerman, David. 2014. "An Introduction to Partition Logic." Logic Journal of the IGPL 22 (1): 94-125.

[13] Ellerman, David. 2015. "Why Delayed Choice Experiments Do NOT Imply Retrocausality." Quantum Studies: Mathematics and Foundations 2 (2): 183-99.

[14] Ellerman, David. 2017. "Logical Information Theory: New Foundations for Information Theory." Logic Journal of the IGPL 25 (5 Oct.): 806-35. https://doi.org/10.1093/jigpal/jzx022.

[15] Ellerman, David. 2017. "Quantum Mechanics over Sets: A Pedagogical Model with NonCommutative finite Probability Theory as Its Quantum Probability Calculus." Synthese 194 (12): 4863-96.

[16] Ellerman, David. 2018. "Logical Entropy: Introduction to Classical and Quantum Logical Information Theory." Entropy 20 (9): Article ID 679. https://doi.org/10.3390/e20090679. 
[17] Ellerman, David. 2018. "The Quantum Logic of Direct-Sum Decompositions: The Dual to the Quantum Logic of Subspaces." Logic Journal of the IGPL 26 (1 January): 1-13. https://doi.org/10.1093/jigpal/jzx026.

[18] Ellerman, David. 2018. "The Objective Indefiniteness Interpretation of Quantum Mechanics." ArXiv.Org. September 2018. http://arxiv.org/abs/1210.7659v2.

[19] Feynman, Richard P. 1985. QED: The Strange Theory of Light and Matter. Princeton NJ: Princeton University Press.

[20] Feynman, Richard P., Robert B. Leighton, and Matthew Sands. 2010. The Feynman Lectures on Physics: Quantum Mechanics Vol. III (New Millennium Ed.). Reading MA: Addison-Wesley.

[21] Finberg, David, Matteo Mainetti, and Gian-Carlo Rota. 1996. "The Logic of Commuting Equivalence Relations." In Logic and Algebra, edited by Aldo Ursini and Paolo Agliano, 69-96. New York: Marcel Dekker.

[22] Fine, Arthur 1986. The Shaky Game: Einstein, Realism, and the Quantum Theory. Chicago: University of Chicago Press.

[23] Heisenberg, Werner. 1962. Physics 83 Philosophy: The Revolution in Modern Science. New York: Harper Torchbooks.

[24] Jaeger, Gregg. 2014. Quantum Objects: Non-Local Correlation, Causality and Objective Indefiniteness in the Quantum World. Heidelberg: Springer.

[25] Shannon, Claude E. 1948. "A Mathematical Theory of Communication." Bell System Technical Journal 27: 379-423; 623-56.

[26] Shapiro, Stewart. 2011. The Company Kept by Cut-Abstraction (and It Relatives). Philosophia Mathematica (III) 19: 107-38.

[27] Shimony, Abner 1988. The reality of the quantum world. Scientific American. 258 (1): 46-53.

[28] Shimony, Abner. 1999. "Philosophical and Experimental Perspectives on Quantum Physics." In Philosophical and Experimental Perspectives on Quantum Physics: Vienna Circle Institute Yearbook 7, 1-18. Dordrecht: Springer Science+Business Media.

[29] Sternberg, Shlomo 1994. Group Theory and Physics. Cambridge: Cambridge University Press.

[30] Stone, Marshall H. 1932. On one-parameter unitary groups in Hilbert Space. Annals of Mathematics. 33 (3): 643-648.

[31] Univalent Foundations Program. 2013. Homotopy Type Theory: Univalent Foundations of Mathematics. Princeton: Institute for Advanced Studies.

[32] Von Neumann, John. 1955. Mathematical Foundations of Quantum Mechanics. Translated by Robert Beyer. Princeton: Princeton University Press.

[33] Weyl, Hermann. 1949. Philosophy of Mathematics and Natural Science. Princeton: Princeton University Press. 